\title{
Birkhoff's HSP-Theorem for Cumulative Logic Programs
}

\author{
Beatrice Amrhein \\ ETH Zürich Universität Tübingen \\ amrhein@math.ethz.ch
}

\begin{abstract}
Birkhoff's HSP theorem is that the models of a set of algebraic equations form a variety, i.e. a category of algebras which admits homomorphic images, subalgebras and products. We show here first, that every equational set of retract structures in combinatory logic is a varriety, and second, that every set of combinators, closed under certain operations, is equational. It follows that the models of cumulative logic programs form an equational variety.
\end{abstract}

\section{Motivation}

The solutions of a set of equations form a structure, which is closed under the operations of building substructures, homomorphic images and products of solutions. This was first shown by G. Birkhoff [5] in 1935 for algebraic equations. We examined here the characterisation of solutions of cumulative logic programs. As in the case of symmetric search trees, where this knowledge can be used to cut symmetric branches, knowledge about the structure of the solutions of a cumulative logic program can speed up their computation.

\section{Cumulative Logic Programs and Combinatory Models}

To perform transformations on arbitrary knowledge bases, (even ones containing rule terms) and to allow more than one argument to such transformations, we have to build a cumulative hierarchy of rules. Therefore, the language of cumulative logic programs was introduced [6], which is based on the language of logic programs with the following extensions: Clauses are written as $\left\{b_{1}, \ldots, b_{k}\right\} \rightarrow h$, where the head $h$ and the body $\left\{b_{1}, \ldots, b_{k}\right\}$ are either atomic formulas or again clauses.

This cumulative formation of rules to an arbitrary level has its mathematical counterpart in graph models $\mathcal{D}_{A}$.

Definition 1. Let $A$ be a set of atomic formulas. $\mathcal{D}_{A}:=\left\langle 2^{G(A)} ; \circ\right\rangle$ is a graph model over $A$, where $G(A)$ is defined recursively:

$$
\begin{array}{ll}
G_{0}(A) & :=A \\
G_{n+1}(A) & :=G_{n}(A) \cup\left\{\alpha \rightarrow a: a \in G_{n}(A), \alpha \subseteq G_{n}(A), \alpha \text { finite }\right\} \\
G(A) & :=\bigcup_{n} G_{n}(A)
\end{array}
$$


Note that within this cumulative hierarchy, pure logic programs are simply subsets of $G_{1}(A)$. The inclusion of implications in goal formulae, as proposed in [9] by Miller et al, leads then to subsets of $G(A)$ of the form

$$
\left\{\alpha_{1} \rightarrow\left(\alpha_{2} \rightarrow\left(\cdots \rightarrow \alpha_{n}\right) \cdots\right), \quad \cdots\right\}
$$

where $\alpha_{i}(1 \leq i \leq n)$ are finite subsets of $A$.

Graph models are rich enough in structure to form models for knowledge bases of arbitrary complexity, where even transformations of functions (such as derivation or fixed point operation) are included as elements. [1]

The application operation $\circ$ is defined as:

$$
V \circ W:=\{a: \alpha \rightarrow a \in V, \alpha \subseteq W\}
$$

The result of applying $V$ to $W$ depends on the elementary instructions $\rho \rightarrow r$ of $V$, which give output $r$ any time the input $W$ contains $\rho$. The application operation can be used to explain the execution of pure logic programs as a solution process for a corresponding fix-point equation as in the case of pure logic programs, the application operation o corresponds to Kowalski's semantics operator $T_{P}$ as defined in [8], i.e. for a combinator $V$ and a set $W$ of ground terms we obtain

$$
V \circ W=T_{P V}(W)
$$

where $P_{V}$ is the logic program derived from combinator $V$.

It's a well-known fact that, these structures $\left\langle 2^{C(A)} ; 0\right\rangle$ build combinatory algebras.

Definition 2. [3] An applicative structure $\mathcal{M}:=\langle M ; \cdot\rangle$ is an algebraic structure with carrier set $M$ and a binary operation , which we call application. By combinatory algebra we denote an applicative structure $\mathcal{M}:=\langle M ; \cdot\rangle$ which is combinatory complete, which means that for every tem $t\left(v_{1}, \ldots, v_{k}\right.$ ) (built. up from elements of $M$ and variables by means of the application and parentheses), an element $T \in M$ exists such that $\forall m_{1}, \ldots, m_{k} \in M$ :

$$
T \cdot m_{1} \cdots m_{k}=t\left(m_{1}, \ldots, m_{k}\right)
$$

Lemma 3 Schönfinkel, Curry. An applicative structure $\mathcal{M}$ is combinatory complete iff there exists $K$ and $S$ such that

$$
\begin{gathered}
\forall v_{1}, v_{2}: K v_{1} v_{2}=v_{1} \\
\forall v_{1}, v_{2}, v_{3}: S v_{1} v_{2} v_{3}=v_{1} v_{3}\left(v_{2} v_{3}\right)
\end{gathered}
$$

In order to present, a readable and comprehensive treatment of the objects in a graph model $D_{A}$ the following notation will be used; 
Variables: $a, b, c, \ldots \quad$ for elements of $\mathrm{A}$

$r, s, t, \ldots$ for elements of $G(A)$

$\rho, \sigma, \tau, \ldots \quad$ for finite subsets of $G(A)$

$V, W \quad$ for elements of $D_{A}$

Terms: $\quad U V W \quad$ for $(U \circ V) \circ W$, i.e. we employ left-associativity and omit o

$\sigma \rightarrow \rho \rightarrow r$ for $\sigma \rightarrow(\rho \rightarrow r)$; right-associativity for arrow terms corresponds to the left-associativity for the application

We define

$$
\begin{aligned}
K:=\{(\sigma \rightarrow(\rho \rightarrow r)): r \in \sigma\} \\
S:=\left\{\left(\tau \rightarrow(\sigma \rightarrow(\rho \rightarrow r)): \exists \rho_{0}, \ldots, \rho_{n} \subseteq \rho, \exists r_{1}, \ldots, r_{n}:\right.\right. \\
\left.\quad \rho_{i} \rightarrow r_{i} \in \sigma(1 \leq i \leq n),\left(\rho_{0} \rightarrow\left(\left\{r_{1}, \ldots, r_{n}\right\} \rightarrow r\right)\right) \in \tau\right\}
\end{aligned}
$$

With this we obtain

Lemma 4. Every graph model is a combinatory algebra.

Definition 5. $\mathcal{M}:=\langle M ; \cdot, K, S, L\rangle$ is a combinatory model if $\mathcal{M}:=$ $\langle M ; \cdot, K, S\rangle$ is a combinatory algebra with combinators $L_{k}(k \in \mathbb{N})$ satisfying the axioms:

$$
\begin{aligned}
\forall u, v_{1}, \ldots, v_{k} \in M & : \quad L_{k} u v_{1} \cdots v_{k}=u v_{1} \cdots v_{k} \\
\forall u_{1}, u_{2} \in M: & \left(L_{k} u_{1}=L_{k} u_{2}\right. \\
& \left.\Leftrightarrow \forall v_{1}, \ldots, v_{k} \in M: u_{1} v_{1} \cdots v_{k}=u_{2} v_{1} \cdots v_{k}\right)
\end{aligned}
$$

As can easily be seen, the graph model combinators

$$
\begin{aligned}
L_{k+1}:=\left\{\rho_{0} \rightarrow(\cdots \rightarrow\right. & \left.\left(\rho_{k} \rightarrow r\right) \cdots\right): \\
& \left.\exists \rho_{i}^{\prime} \subseteq \rho_{i}(1 \leq i \leq k), \rho_{1}^{\prime} \rightarrow\left(\cdots \rightarrow\left(\rho_{k}^{\prime} \rightarrow r\right) \cdots\right) \in \rho_{0}\right\}
\end{aligned}
$$

have the above mentioned properties (1) and (2), which means that $\mathcal{D}_{A}$ is indeed a combinatory model.

When querying a Prolog program, the resolution mechanism implicitly searches for the least fixed point of a program equation $v=F \cdot v$. By combinatory completeness, we can state a combinator $Y$ with the property:

$$
Y V=V(Y V)
$$

which guarantees solutions for every fix-point program-equation in any graph model.

Equations of cumulative logic programs are solved explicitly using the algorithms formulated in [10] in the case of fix-point equations, or in more general cases using the semi-algorithm as sketched in [7]

A problem is in general a set of equations $\langle a, b\rangle$ where $a$ and $b$ are combinators (programs). Solutions of a problem are, therefore, again elements of the graph model, which means, again cumulative logic programs. In general we get an (program-)approximation to a solution in the sense of [6], in more special cases the solutions can be found in the ground set $A[10]$. 


\section{Definitions}

In the original Theorem of Birkhoff, the signature of the algebras has to be fixed to define the language of the equations. In an analogous way, we first have to determine the language of the considered equations.

Definition 6. Let $\mathcal{M}$ be a combinatory model. Let $\Theta:=\left\{\theta_{j}: j \in J\right\}$ be a set of distinguished combinators in $\mathcal{M}$. $s$ is called $\Theta$-term if $s \in \Theta, s=K$ or $s=S$, and if $s_{1}, s_{2}$ are $\Theta$-terms, then $\left(s_{1} s_{2}\right)$ is also a $\Theta$-term.

A pair $\langle s, t\rangle$ is called $\Theta$-equation, if $s$ and $t$ are $\Theta$-terms, or if there is a $k \in \mathbb{N}$ with $s=L_{k} s^{\prime}$ and $t=L_{k} t^{\prime}$, where $s^{\prime}$ and $t^{\prime}$ are $\Theta$-terms.

Because of combinatory completeness, every term $t$ can be written in the form $t^{\prime} v_{1} \cdots v_{k}$, where $v_{1}, \ldots, v_{k}$ are the variables contained in $t$. We will, therefore, restrict our attention to $\Theta$-equations.

In the first section, retractions will play the role of the solution sets (as do algebras in the original theorem of Birkhoff).

Definition 7. Let $\mathcal{M}$ be a combinatory model. A combinator $x$ in $\mathcal{M}$ is called retraction, if for all $v \in \mathcal{M}$ :

$$
x(x v)=x v
$$

With retract of $x$ we denote the set $\operatorname{ret}(x):=\{v \in M: x v=v\}$

We can now build restricted quantifier eliminators over retractions. For an arbitrary retraction $x$ we denote by

$$
L^{x}:=B L(C B x), \quad \text { and } \quad L_{k}^{x}:=B(B L(C B x))\left(B L_{k}^{x}\right)
$$

where $B$ and $C$ are combinators defined as:

$$
\forall v_{1}, v_{2}, v_{3}: B v_{1} v_{2} v_{3}=v_{1}\left(v_{2} v_{3}\right)
$$

and

$$
\forall v_{1}, v_{2}, v_{3}: C v_{1} v_{2} v_{3}=v_{1} v_{3} v_{2}
$$

Then, we can show:

Lemma 8. For any retraction $x, L^{x}$ is a normalising combinator for the retract of $x$, which means that for all $k \in \mathbb{N}$ :

$$
\begin{gathered}
\forall u, v_{1}, \ldots, v_{k}: L_{k}^{x} u v_{1} \cdots v_{k}=u\left(x v_{1}\right) \cdots\left(x v_{k}\right) \\
\forall u_{1}, u_{2}\left(\forall v_{1} \cdots v_{k}: u_{1}\left(x v_{1}\right) \cdots\left(x v_{k}\right)=u_{2}\left(x v_{1}\right) \cdots\left(x v_{k}\right) \Leftrightarrow L_{k}^{x} u_{1}=L_{k}^{x} u_{2}\right)
\end{gathered}
$$


By these restricted quantifier eliminators, we can also eliminate variables in equations like $s\left(x v_{1}\right)=t\left(x v_{1}\right)\left(x v_{2}\right)$, because:

$$
\begin{aligned}
s\left(x v_{1}\right)=t\left(x v_{1}\right)\left(x v_{2}\right) & \Leftrightarrow K\left(s\left(x v_{1}\right)\right)\left(x v_{2}\right)=t\left(x v_{1}\right)\left(x v_{2}\right) \\
& \Leftrightarrow K\left(s\left(x v_{1}\right)\right)\left(x v_{2}\right)=t\left(x v_{1}\right)\left(x v_{2}\right) \\
& \Leftrightarrow B K s\left(x v_{1}\right)\left(x v_{2}\right)=t\left(x v_{1}\right)\left(x v_{2}\right) \\
& \Leftrightarrow L_{2}^{x}(B K s)=L_{2}^{x} t
\end{aligned}
$$

which means we can restrict our attention on equations without quantifiers.

The examined equations will be over a fixed language $\mathcal{L}:=\Theta \cup\{K, S\}$ and variables, which will be eliminated by $L$-combinators:

Definition 9. A retraction $x$ is called a solution of a $\Theta$-equation $\left\langle L_{k} s, L_{k} t\right\rangle$, if $L_{k}^{x} s=L_{k}^{x} t$ which is equivalent to:

$$
\forall v_{1}, \ldots, v_{k}: x\left(s\left(x v_{1}\right) \cdots\left(x v_{k}\right)\right)=x\left(t\left(x v_{1}\right) \cdots\left(x v_{k}\right)\right)
$$

or literally, a retraction $x$ is a solution of $\left\langle L_{k} s, L_{k} t>\right.$ if the following holds: for all $v_{1}, \ldots, v_{k} \in \operatorname{ret}(x)$, if $s v_{1} \cdots v_{k} \in \operatorname{ret}(x)$ and $t v_{1} \cdots v_{k} \in \operatorname{ret}(x)$ then $s v_{1} \cdots v_{k}=t v_{1} \cdots v_{k}$ must hold.

Example. Let $s$ and $t$ be combinators of the form:

$s:=\{\emptyset \rightarrow a,\{v\} \rightarrow(\{v\} \rightarrow v): a \in A\}$

$t:=\{\{\alpha\} \rightarrow \alpha: \alpha \subseteq G(A), \alpha$ finite $\}$

A retraction $x$ is then a solution of $\langle s, t\rangle$ if the elements of ret.( $x)$ are of the form $\{\{a\},\{\{a\} \rightarrow a\}, \ldots\}$.

Note 10. To keep the formulas readable, we introduce the following abbreviation: Let $x$ be a retraction, $s$ a combinator and $v_{1}, \ldots, v_{k}$ any variables. Then, we write $s(\overline{x v})$ for $s\left(x v_{1}\right) \cdots\left(x v_{k}\right)$.

\section{Birkhoff's Theorem for Retract Equations}

Our target will be to show Birkhoff's theorem for equations with retract-solutions in a combinatory model $\mathcal{M}$. Solutions of such equations are originally algebras or (relational) structures of some special type. Our approach will be different: we examine equations whose solutions are retractions. These retractions are not usually closed under $\Theta$-terms, which means that solutions need not be structures in the usual sense.

In [11] it is shown how to represent any algebraic or relational structure in a combinatory model using retractions. Our approach is therefore a generalisation of Birkhoff's original theorem.

Assume $x$ is a retract solution of some set of equations $\Gamma$. First, we investigate the question of how to find new solutions of $\Gamma$ from solutions $x$. For this we define subretractions, homomorphic images and products of retractions. 
Definition 11. $y$ is called a $\Theta$-subretraction of $x$, if

$$
\begin{aligned}
& \forall v: y(y v)=y v \\
& \forall v: x(y v)=y v
\end{aligned}
$$

and for all $\theta_{j} \in \Theta, \forall v_{1}, \ldots, v_{k}$ :

$$
x\left(\theta_{j}(\overline{y v})\right)=y\left(\theta_{j}(\overline{y v})\right)
$$

$\Theta$-subretractions are retractions (3), which define subsets of the retract of $x$ (4) and which preserve $\Theta$-equations (5). Hence, as can easily be observed, they satisfy the following lemma:

Lemma 12. Let $x$ be a retraction and $y$ a $\Theta$-subretraction of $x$. If $x$ is a solution of some $\Theta$-equation $\left\langle L_{k} s, L_{k} t>\right.$, then $y$ is a solution too.

Proof. $\forall v_{1}, \ldots, v_{k}$ :

$$
\begin{aligned}
& x(s(\overline{x v}))=x(t(\overline{x v})) \\
& \quad \Rightarrow x(s(x(\overline{y v})))=x(t(x(\overline{y v}))) \\
& \quad \Rightarrow x(s(\overline{y v}))=x(t(\overline{y v})) \\
& \quad \Rightarrow y(s(\overline{y v}))=y(s(\overline{y v}))
\end{aligned}
$$

In a next step we define $\Theta$-homomorphisms and show that $\Theta$-homomorphic images of a solution $x$ are also solutions:

Definition 13. Let $x$ and $y$ be retractions in $\mathcal{M}$ and $\varphi \in M$ a combinator. $\varphi$ is called a $\Theta$-homomorphism from $x$ to $y$ if for all $\theta \in \Theta$ :

$$
y(\theta(\overline{\varphi(x v)}))=y(\varphi(\theta(\overline{x v})))
$$

$\varphi$ is called a $\Theta$-epimorphism, if for all $\theta_{i}, \theta_{j} \in \Theta$ :

$$
\begin{array}{r}
\forall v_{1}, \ldots, v_{k}: \quad y\left(\theta_{i}(\overline{\varphi(x v)})\right)=y\left(\theta_{j}(\overline{\varphi(x v)})\right) \\
\Rightarrow y\left(\theta_{i}(\overline{y v})\right)=y\left(\theta_{j}(\overline{y v})\right)
\end{array}
$$

A combinator $\varphi$ is a $\Theta$-homomorphism if it is compatible with $\Theta$-combinators in $\operatorname{ret}(y)(6)$. However, to be a $\Theta$-epimorphism, $\varphi$ needs not, be onto ret $(y)$.

With these definitions we can show:

Lemma 14. Let $x$ and $y$ be retractions and $\varphi$ a $\Theta$-epimorphism from $x$ to $y$. If $x$ is a solution of a $\Theta$-equation $\left\langle L_{k} s, L_{k} t\right\rangle$, then so is $y$.

Proof. Assume $\boldsymbol{x}$ is a solution of some $\Theta$-equation $\left\langle L_{k} s, L_{k} t\right\rangle$, so by definition of $L$ holds for all $v_{1}, \ldots, v_{k}$ :

$$
x(s(\overline{x v}))=x(t(\overline{x v}))
$$

and

$$
y(\varphi(s(\overline{x v})))=y(\varphi(s(\overline{x v})))
$$

As $\varphi$ is a $\Theta$-homomorphism, we can infer 


$$
y(s(\overline{\varphi(x v)}))=y(s(\overline{\varphi(x v)}))
$$

which is equivalent to

$$
L_{k}^{B \varphi x}\left(B^{k} y s\right)=L_{k}^{B \varphi x}\left(B^{k} y t\right)
$$

and by (7)

$$
L_{k}^{y}\left(B^{k} y s\right)=L_{k}^{y}\left(B^{k} y t\right)
$$

we conclude that $y$ is also a solution.

A third possibility to find new solutions is by forming products of given solutions.

Definition 15. Let $J$ and $\{x(J j): j \in M\}$ be retractions. $y$ is called a $\Theta-p r o-$ duct of $\{x(J j): j \in M\}$ if the following equations are satisfied:

for all $v$ :

$$
y(y v)=y v
$$

for all $\theta \in \Theta$ and all $v_{1}, \ldots, v_{k}$ :

$$
\begin{aligned}
& y\left(\theta\left(y v_{1}\right) \cdots\left(y v_{k}\right)\right)(J j) \\
& \quad=x(J j)\left(\theta\left(x(J j)\left(v_{1}(J j)\right)\right) \cdots\left(x(J j)\left(v_{k}(J j)\right)\right)\right)
\end{aligned}
$$

and

$$
L^{J}\left(y\left(\theta\left(\overline{y^{v}}\right)\right)\right)=y(\theta(\overline{y v}))
$$

To be a $\Theta$-product of a given set of retractions, $y$ itself has to be a retraction (8). The equations in (9) and (10) confirm that the $\Theta$-terms in the projections $y(J j)$ of $y$ have the same normal form as those in $x(J j)$. These conditions are sufficient to satisfy the following lemma.

Lemma 16. Let $\{x(J j): j \in M\}$ be a set of solutions of some $\Theta$-equation $<L_{k} s, L_{k} t>$. If $y$ is a $\Theta$-product of $\{x(J j): j \in M\}$ then $y$ is also a solution.

Proof. Let $<L_{k} s, L_{k} t>$ be a $\Theta$-equation such that $\{x(J j): j \in M\}$ are solutions of it. Hence:

$$
\begin{aligned}
& \forall j \in \operatorname{ret}(J), \forall v_{1}, \ldots, v_{k}: \\
& \quad(x j)\left(s\left((x j)\left(v_{1} j\right)\right) \cdots\left((x j)\left(v_{k} j\right)\right)\right)=(x j)\left(t\left((x j)\left(v_{1} j\right)\right) \cdots\left((x j)\left(v_{k} j\right)\right)\right)
\end{aligned}
$$

By equation (9) we can infer for all $v_{1}, \ldots, v_{k}$ :

$$
\forall j: \quad y(s(\overline{y v}))(J j)=y(t(\overline{y v}))(J j)
$$

By definition of $L^{J}$ this is equivalent to:

$$
L^{J}(y(s(\overline{y v})))=L^{J}(y(t(\overline{y v})))
$$

and as the $\Theta$-terms are in normal form over $J$ we conclude:

$$
y(s(\overline{y v}))=y(s(\overline{y v}))
$$

thus, the product $y$ is also a solution of $\left\langle L_{k} s, L_{k} t\right\rangle$. 


\subsection{Proof of a HSP-Theorem for Retract-Solutions}

Now, we have found operations, which preserve $\Theta$-equations. It is much more difficult, however, to ascertain whether or not these three operations $(\Theta$-subretraction, $\Theta$-homomorphism, $\Theta$-product) are sufficient to construct all solutions of a given set of equations. The following theorem provides an answer:

Theorem 17. Let $\mathcal{M}:=\langle M ; \cdot, K, S, L\rangle$ be a combinatory model. Given a set $\mathcal{K}:=\{x i: i \in I\}$ of retractions in $M, \mathcal{K}$ is equational iff $\mathcal{K}$ is closed under $\Theta$-subretractions, $\Theta$-epimorphisms and $\Theta$-products.

Proof. We will split the proof into lemmas 18 and 19 . Let $\Gamma\left(x_{i}\right)$ be the set of $\Theta$-equations, of which $x_{i} \in \mathcal{K}$ is a solution. We define $\Gamma(\mathcal{K}):=\cap_{i \in I} \Gamma\left(x_{i}\right)$. Let $y$ be a solution of $\Gamma(\mathcal{K}) . M$ is not empty, so is ret $(y)$ either, because for all $v$ in $M, y v$ is an element of ret $(y)$.

We define $\Psi$ to be the following set of combinators:

$$
\Psi:=\{\varphi: \varphi(y v)=x(\varphi(y v))\}
$$

We choose a retraction $\chi$ with the property $|\operatorname{ret}(\chi)| \geq|\Psi|$. Without loss of generality, we can assume that $\chi$ is an enumeration of $\Psi$ so $\Psi=\left\{\varphi_{i}: i \in \chi\right\}$. Let $x^{x}$ be a power of $x$. We then define the following combinator:

$$
\phi(y u)\left(\chi^{i}\right):=\varphi_{i}(y u)
$$

In a next step we describe the image of $\phi$ in $x^{x}$ : Let $v_{0}$ be an element of $\operatorname{ret}(y)$.

$$
z u:= \begin{cases}x^{\chi} u & \exists v, u=\phi(y v) \\ x^{\chi}(\theta(\overline{z v})) \quad \exists v_{1}, \ldots, v_{k}, u=x^{\chi}(\theta(\overline{\phi(y v)})) \\ x^{\chi}\left(\phi\left(y v_{0}\right)\right) \text { else }\end{cases}
$$

Lemma 18. $z$ is a $\Theta$-subretraction of $x^{\chi}$.

Proof. We first show that $z$ is a retraction:

$$
z(z u)= \begin{cases}z\left(x^{\chi} u\right) & \exists v, u=\phi(y v) \\ z\left(x^{\chi}(\theta(\overline{\phi(y v)}))\right) & \text { or } \\ z\left(x^{\chi}\left(\phi\left(y v_{0}\right)\right)\right) & \text { else }\end{cases}
$$

As $x^{\chi}(\phi(y v))=\phi(y v)$, the assumption is clear.

In the second step, we show that $z$ is a $\Theta$-subretraction. We have to show that for all $\theta \in \Theta$ and all $v_{1}, \ldots, v_{k}$ :

$$
\begin{gathered}
x^{X}\left(\theta\left(z v_{1}\right) \cdots\left(z v_{k}\right)\right)=z\left(\theta\left(z v_{1}\right) \cdots\left(z v_{k}\right)\right) \\
z\left(\theta\left(z v_{1}\right) \cdots\left(z v_{k}\right)\right)=z\left(\theta\left(\phi\left(y w_{1}\right)\right) \cdots\left(\phi\left(y w_{k}\right)\right)\right) \text { for some } w_{1}, \ldots, w_{k} .
\end{gathered}
$$




$$
\begin{aligned}
z\left(\theta\left(\phi\left(y w_{1}\right)\right) \cdots\left(\phi\left(y w_{k}\right)\right)\right) & =z\left(\phi\left(\theta\left(y w_{1}\right) \cdots\left(y w_{k}\right)\right)\right) \\
& =x^{\chi}\left(\phi\left(\theta\left(y w_{1}\right) \cdots\left(y w_{k}\right)\right)\right) \\
& =x^{\chi}\left(\theta\left(\phi\left(y w_{1}\right)\right) \cdots\left(\phi\left(y w_{k}\right)\right)\right) \\
& =x^{\chi}\left(\left(z v_{1}\right) \cdots\left(z v_{k}\right)\right)
\end{aligned}
$$

It remains to be shown that $y$ is a homomorphic image of the retraction $z$. We define:

$$
\psi(z u):= \begin{cases}y v & \exists v, u=\phi(y v) \\ y(s(\overline{\psi(z v)})) & \exists v_{1}, \ldots, v_{k}, \exists s \in \theta u=x^{\chi}(s(\overline{z v}))\end{cases}
$$

Lemma 19. $\psi$ is a $\Theta$-epimorphism from $z$ to $y$.

Proof. Let $u$ and $v$ be combinators in ret $(y)$ with $u \neq v$. By definition of $\phi$ we can also infer that $\phi v \neq \phi u$, which means that $x^{\chi}(\phi v) \neq x^{\chi}(\phi u)$.

Assume that $y(s(\overline{y v})) \neq y(t(\overline{y v}))$ for some $\Theta$-terms $L_{k} s$ and $L_{k} t$. As $y$ is a solution of $\Gamma(\mathcal{K})$ we can also infer that $x^{\chi}\left(s\left(\overline{x^{\chi} v}\right)\right) \neq x^{\chi}\left(t\left(\overline{x^{\chi} v}\right)\right)$, hence $z(s(\overline{z v})) \neq z(t(\overline{z v}))$, from where we can infer, that $\psi$ is well defined. Obviously, $\psi$ is also a $\Theta$-epimorphism.

This completes the proof for Theorem 17, as we have constructed a $\Theta$-subretraction $z$ of a $\Theta$-product $x^{\chi}$ and a $\Theta$-homomorphism $\psi$ from $z$ to $y$.

\section{Birkhoff's Theorem for Combinator Solutions}

In a second approach, we drop the condition that solutions have to be retractions, i.e. every combinator (or every cumulative logic program) is admitted as a solution (or as an approximation).

The intended meaning of such a solution (set) is the following: Let $x$ be any combinator, a combinator $a$ is an element of a set $\tilde{x}$ if $a$ can be written as $a=a^{\prime} x$. Or to put it simply:

$$
\tilde{x}:=\{a x: a \in D\}
$$

This gives a generalization of the retract-construction we used in the last, section. Let $x$ be any retraction, then $x a$ is an element of the retract of $x$. However, $x a$ can be written as $x a=C I a x$ and because $a^{\prime}=C I a, a$ is shown to be an element of $\tilde{x}$.

Therefore, the equations we examine will be of the form $\langle s, t\rangle$, where a combinator $x$ is a solution of this equation, if $x$ satisfies $s x=t x$. 
As in the previous section, we first try to find operations which preserve $\Theta$ equations. Roughly speaking, these are multiplications from the right and from the left. The strength of combinatory logic allows us to deduce some results from these two operations already.

Definition 20. Let $\Theta$ be a set of distinguished combinators and $\Gamma$ any set of $\Theta$-equations, i.e.

$$
\Gamma \subseteq\{\langle s, t\rangle: s, t \Theta \text {-terms }\}
$$

Now, a combinator $x$ is a solution of $\Gamma$ if $x$ satisfies $s x=t x$ for every pair $<s, t>$ in $\Gamma$.

This is a more general definition than the one we had before, because for every term of the form $x(s(\overline{x v}))$ we can (by combinatory completeness) find a term of the form $s^{\prime} x$, which is equivalent to $x(s(\overline{x v}))$.

If $s x=t x$ then for all combinators $\nu$ and $\mu, \nu(s x) \mu=\nu(t x) \mu$ holds. This leads to the definition of $\Theta$-restrictions.

Definition 21. $y$ is called a $\Theta$-restriction of $x$ if there are combinators $\nu, \mu$ so that for all $\Theta$-terms $s$ :

$$
s y=\nu(s x) \mu
$$

Obviously, the following lemma is true:

Lemma 22. If a combinator $x$ is a solution of a set of equations $\Gamma$, then every restriction of $x$ is a solution, too.

Proof. Let $\langle s, t\rangle$ be a $\Theta$-equation and assume $x$ to be a solution of this equation. Then:

$$
\begin{aligned}
s x=t x & \Rightarrow \forall \nu, \nu(s x)=\nu(t x) \\
& \Rightarrow \forall \nu, \mu \nu(s x) \mu=\nu(t x) \mu \\
& \Rightarrow s y=t y
\end{aligned}
$$

$x$ is always a $\Theta$-restriction of itself. We can show this by choosing $\nu=\mu=K$, because for all $s, K(s x) K=s x$.

We will call a combinator $\varphi$ homomorphic on $x$ if $\varphi$ commutes on the $\Theta$ elements of $x$.

Definition 23. A combinator $\varphi$ is a $\Theta$-homomorphism on $x$ if $\varphi$ satisfies for all $\Theta$-terms $u$ :

$$
\varphi(u x)=u(\varphi x)
$$

$\varphi x$ is then called the $\Theta$-homomorphic image of $\varphi$. 
Every homomorphism on $x$ leads to a $\Theta$-restriction of $x$. Because $\varphi(s x)$ is equal to $K(\varphi(s x)) I$ for all $\Theta$-terms $s$, and as $K(\varphi(s x)) I$ is equal to $B K \varphi(s x) I$, we can choose $\nu=B K \varphi$ and $\mu=I$ and obtain $s(\varphi x)=\nu(s x) \mu$.

Therefore, we can state:

Lemma 24. If $y$ is the homomorphic image of a combinator $x$ (and of a $\Theta$. homomorphism $\varphi$ ), then $y$ is a $\Theta$-restriction of $x$.

Lemma 25. Let $x$ be a solution of any $\Theta$-equation $\langle s, t\rangle$ and $\varphi$ a $\Theta$-homomorphism on $x$. If $y=\varphi x$ is the homomorphic image of $\varphi$, then $y$ is a solution of $\langle s, t\rangle$.

Proof. Let $s$ and $t$ be any $\Theta$-terms such that $x$ is a solution of $\langle s, t\rangle$.

$$
\begin{aligned}
s x=t x & \Rightarrow \varphi(s x)=\varphi(t x) \\
& \Rightarrow s(\varphi x)=t(\varphi x) \\
& \Rightarrow s y=t y
\end{aligned}
$$

We will call a combinator $y$ a product of a set of combinators $\{x j: j \in J\}$, if its projections $(y j), j \in J$ behave on $\Theta$-terms the same way as $(x j), j \in J$.

Definition 26. Let $J$ be any retraction and $\{x j: j \in J\}$ a set of combinators in $D . y$ is called a $\Theta$-product of $\{x j: j \in J\}$ if for all $\Theta$-terms $s$ :

$$
L^{J}(s y)=s y
$$

and

$$
L^{J}(s y)=L^{J}(B s x)
$$

These two conditions are sufficient to show the following lemma:

Lemma 27. If $\{x j: j \in J\}$ are solutions of any $\Theta$-equation $\langle s, t\rangle$ and $y$ is a $\Theta$-product of $\{x j: j \in J\}$, then $y$ is also a solution of $\langle s, t\rangle$.

Proof. Assume $y$ to be a $\Theta$-product of the set of combinators $\{x j: j \in J\}$, and $s$ and $t$ two $\Theta$-terms, so that for all $j$ in $J, x j$ is a solution of $\langle s, t\rangle$ :

$$
\begin{aligned}
& \forall j: s(x(J j))=t(x(J j)) \\
& \Rightarrow \forall j:(s y)(J j)=(t y)(J j) \\
& \Rightarrow L^{J}(s y)=L^{J}(t y) \\
& \Rightarrow s y=t y
\end{aligned}
$$

because by equation (12) $s y$ and $t y$ are in normal form. 


\section{Proof of the HSP-Theorem}

We have shown that every $\Theta$-restriction, every $\Theta$-homomorphic image, and every $\Theta$-product of solutions of $\Theta$-equations are new solutions. In the next theorem we will show that the converse is also true. We begin with some definitions.

Let $\tilde{\Theta}$ be the set of $\Theta$-terms and $x$ a combinator. We define $\Phi$ to be the following set of combinators:

$$
\begin{aligned}
\Phi_{x} & :=\left\{\varphi_{j}: \exists \nu_{j}, \mu_{j}, \forall s \in \tilde{\Theta}, \varphi_{j} s=\nu_{j}(s x) \mu_{j}\right\} \\
& =\left\{\varphi_{j}: \varphi_{j}=C\left(B\left(C \nu_{j} \mu_{j}\right)\right) x, \nu_{j}, \mu_{j} \in D\right\}
\end{aligned}
$$

Let $\kappa$ be a retraction with $|\operatorname{ret}(\kappa)| \geq\left|\Phi_{x}\right|$.

For $j \in \kappa$ let $z j$ be the image of $\tilde{\Theta}$ under $\varphi_{j}$, i.e.

$$
s z(\kappa j):=\varphi_{j} s \quad\left(=\nu_{j}(s x) \mu_{j}\right)
$$

Some of the $\varphi_{j}$ may occur more than once if $|\operatorname{ret}(\kappa)|>\left|\Phi_{x}\right|$.

Lemma 28. Let $\mathcal{K}:=\left\{x_{j}: j \in \kappa\right\}$ be a set of combinators in $D, \Gamma$ the set of $\Theta$-equations which are satisfied by $\mathcal{K}$ and $x$ the $\Theta$-product of $\mathcal{K}$. Let $\Phi_{x}$ and $z j$ be the combinators as defined above and $\bar{z}$ the $\Theta$-product of these $z j, j \in \kappa$. Every solution $y$ of $\Gamma$ is then a $\Theta$-homomorphic image of $\bar{z}$.

Proof. Define $\psi$ as the following homomorphism:

$$
\psi(s \bar{z}):=s y \quad(=s(\psi z))
$$

We have to show that $\psi$ is well defined.

Assume $s \bar{z}=t \bar{z}$ for any $\Theta$-terms $s$ and $t$. By definition of $\bar{z}$ we fincl:

$$
\begin{aligned}
s \bar{z}=t \bar{z} & \Rightarrow \forall j: s \bar{z}(\kappa j)=t \bar{z}(\kappa j) \\
& \Rightarrow \forall j:\left(\nu_{j}\right)(s x)\left(\mu_{j}\right)=\left(\nu_{j}\right)(t x)\left(\mu_{j}\right)
\end{aligned}
$$

Now, assume $s x \neq t x$. We choose $\varphi_{i}$ with $\nu=\mu=K$ and find $\nu(s x) \mu \neq$ $\nu(t x) \mu$. We can then infer $s \bar{z} \neq t \bar{z}$, but this is a contradiction.

So $s x=t x$ must be true and, furthermore, $s y=t y$ also, as $y$ is a solution of $\Gamma$. So $\psi$ is well defined.

Finally, we can show:

Theorem 29. Let $\mathcal{D}:=\langle D ; \cdot, K, S, L\rangle$ be a combinatory model and $\mathcal{K}:=\left\{x_{j}:\right.$ $j \in J\}$ a set of combinators which is closed under $\Theta$-restrictions, $\Theta$-products and $\Theta$-homomorphic images.

Let $\Gamma$ be the set of $\Theta$-equations which are satisfied by all of these combinators in $\mathcal{K}$. If $y$ is a solution of the equations $\Gamma$, then $y$ is an element, of $\mathcal{K}$.

Proof. Let $x$ be a $\Theta$-product of $\left\{x_{j}: j \in J\right\}$. Defining $\bar{z}$ the same way as above, every $z(\kappa j)$ is a $\Theta$-restriction of the product $x$. So $\bar{z}$ is a $\Theta$-product of $\Theta$-restrictions of $x$.

$y$ is then the $\Theta$-homomorphic image of $\bar{z}$ where the homomorphism $\psi$ is defined as before. 


\section{References}

[1] K. Aberer, Combinatory differential fields and constructive analysis. PhD thesis ETH No. 9357, ETH Zürich, (1991).

[2] B. Amrhein, Universal Algebra in Combinatory Logic. PhD thesis ETH No. 10005, ETH Zürich, (1992).

[3] H.P. Barendregt. The Lambda Calculus. North Holland, Amsterdain, (1984).

[4] M. J. Beeson. Foundations of Constructive Mathematics. Springer, (1984).

[5] G. Birkhoff. On the Structure of Abstract Algebras. Proc. Cambridge Plil. Soc. 31, (1935).

[6] E. Engeler. Cumulative Logic Programs and Modelling. Logic Collocuium '86, North Holland, pp. $83-93$, (1988).

[7] E. Engeler. Sketch of a New Discipline of Modelling. Report Math. Dept. ETH Zürich, (1988).

[8] J. W. Lloyd. Foundations of Logic Programming. Springer (1987).

[9] J. Hodas, D. Miller, Logic Programming in a Fragment of Intuitionistic Linear Logic, Logics in Computer Science, Amsterdam, (1991).

[10] G. Schwärzler Knowledge-Based Modelling of Cooperative Processes. PhD thesis ETH No. 9926, ETH Zürich (1992).

[11] T. Weibel, Extension of Combinatory Logic to a Theory of Combinatory Representation, TCS, 97: 157-173, (1992). 